\title{
The Illumination Model of the Valley Based on the Diffuse Reflect of Forest
}

\author{
Guoliang $\mathrm{He}^{\mathrm{a}}$, Jiashi Zhou, Cheng $\mathrm{He}$ and Zonghui Xie \\ University of Electronic Science and Technology of China, 611731 Chengdu, China
}

\begin{abstract}
In this paper, models are build to evaluate the impact of the forest on the valley's illumination. Based on the assumes that all the light reach the ground comes from the diffuse reflection which comes from the sun directly and from the diffuse reflection of other points, One model is build to consider the impact of time and latitude on the direction of the sunlight. So we can get the direction of the sunlight at different time and latitude through the model. Besides, this paper develops a illumination model to evaluate the intensity of illumination of the ground. Combining the models above, this paper get a complete model which can not only evaluate the overall light intensity of the valley but also convert the light intensity to the intensity of illumination. Simulation of the intensity illumination of some basic terrains and finally gives a comprehensive results which is practical and close to the common sense.
\end{abstract}

\section{Introduction}

It is known that in mountainous terrain, the brightness of the valley is generally lower than that of the hillside and the mountaintop which attribute to the shelter of trees and mountain peaks. Because of the various of the terrain, the latitudes, the time and the other factors, the intensity of illumination will be different from place to place.

As we all know, the intensity of illumination in the valley is not decided by the sunlight directly. That is to say, because of the diffuse reflection, the area without the sunlight is not necessarily all black. We therefore divide the influence factors into three aspects.

Firstly, we consider the impact of altitude on the intensity of illumination. Although the illumination is not influenced by the altitude directly, the altitude imposes a dramatic effect on the direction of the sunlight. We build the light directions model to contact the latitude and the intensity illumination. So the direction of sunlight finally determines the amount of incoming light.

Secondly, we consider the shelter of the forest. Here we develop a reflection model. In this model, the light from the sum contains two parts. One go through trees and the other reflect to the sky by trees. We will calculate the light reach the land to evaluate the light intensity of the valley.

Thirdly, we know that the density of trees varies with the slope of the mountain and there is also a great relationship between the diffuse reflection coefficient and the density of the tree. So we will build two models: One discuss the relationship between the diffuse reflection coefficient and the density of the tree while the other discuss the relationship between tree density and slope gradient.

Later on, we will combine the models above so as to get a complete model which can not only evaluate the

\footnotetext{
$\overline{{ }^{a} \text { Corresponding author: hegl@uestc.edu.cn }}$
}

overall light intensity of the valley but also convert the light intensity to the intensity of illumination.

At last, after analysis the impact of these factors, some propagation simulation of real situation can be carried out. We will use our models to simulate the intensity illumination of some basic terrains to assess the accuracy and practicality of our model. Furthermore, we can get a comprehensive simulation results which is practical and close to the common sense.

In order to establish the mathematics model easily, we introduce the following assumptions. In the paper, No consideration of the height of the tree. Generally speaking, comparing to the height of the mountain, the height of trees is so small that we treat them as a belt of trees with the same height and all kinds of terrains are evenly covered with trees. Besides, to simplify the question, we assumed that the shelter of the atmosphere has the same effect on the intensity of illumination everywhere. Namely, trees in the valley is close enough and entered no ray of light. So the ground of the valley can not receive light from the sun directly.

\section{Theoretic analysis}

\subsection{Model Direction of Sunlight}

Because the direction of the sunlight varies with the latitude and the time, firstly we have to create a coordinate system and build a model to discuss the relationship between the direction of the sunlight and the latitude as well as the local time.

Here is a glossary of specific audio terminology in our model ${ }^{[1]}$.

(1)Solar time $t_{s}$ : Solar time is a reckoning of the passage of time based on the sun's position in the sky. The fundamental unit of solar time is the day. Two types 
of solar time are apparent solar time (sundial time) and mean solar time (clock time).

China adopts the mean solar time of longitude 120 degrees east as the standard time for the whole country, namely "Beijing time". The latitude and longitude of Beijing is $(39.9 \mathrm{~N}, 116 \mathrm{E})$, showing that "Beijing time" is not the mean solar time of local Beijing. The longitude differ from the true mean solar time about 5 degrees, 20 minutes. The accurate mean solar time can be calculate as follows:

$$
t_{s}=t_{b}+\frac{\varphi-120}{15}
$$

where $t_{b}$ is the Beijing time, $\varphi$ is the local latitude.

(2)Solar hour angle $\omega$ : Observing the sun from earth, the solar hour angle is an expression of time, expressed in angular measurement, usually degrees, from solar noon. At solar noon the hour angle is 0.0 degrees, with the time before solar noon expressed as negative degrees, and the local time after solar noon expressed as positive degrees. For example, at 10:30 AM local apparent time the hour angle is $-22.5^{\circ}\left(15^{\circ}\right.$ per hour times 1.5 hours before noon $)$. So the solar hour angle is defined as:

$$
\omega=15\left(t_{s}-12\right) \text {. }
$$

(3) Solar declination $\delta$ : The angle between the equator and the place on Earth where the sun is straight above you is called the "solar declination", The approximation value is given by

$$
\delta=23.45 \sin \left(\frac{2 \pi(284+n)}{365}\right) .
$$

(4)The solar elevation angle $\alpha$ : The solar elevation angle is the altitude of the sun, the angle between the horizon and the centre of the sun's disc. The approximate value can be calculated with the following formula:

$$
\sin \alpha=\sin \varphi \cdot \sin \delta+\cos \varphi \cdot \cos \delta \cdot \cos \omega
$$

where

$h$ is the hour angle, in the local solar time,

$\delta$ is the current declination of the Sun,

$\varphi$ is the local latitude.

(5)The solar azimuth angle $A$ : The solar azimuth angle is the azimuth angle of the sun.It defines in which direction the sun is, whereas the solar zenith angle or its complementary angle solar elevation defines how high the sun is. There are several conventions for the solar azimuth, however it is traditionally defined as the angle between a line due south and the shadow cast by a vertical rod on Earth. This convention states the angle is positive if the line is east of south and negative if it is west of south The solar azimuth angle can be calculated to a good approximation with the following formula, however angles should be interpreted with care because the inverse sine, i.e. $x=\sin ^{-1}(y)$, has multiple solutions, only one of which will be correct. So we have

$$
\sin A=\frac{-\sin \omega \cdot \cos \delta}{\cos \alpha} .
$$

From the above calculation formula, we can have a systems of equations as follows:

$$
\left\{\begin{array}{l}
t_{s}=t_{b}+\frac{\phi-120}{15} \\
\omega=15\left(t_{s}-12\right) \\
\delta=23.45 \sin \left(\frac{2 \pi(284+n)}{365}\right) \\
\sin \alpha=\sin \phi \cdot \sin \delta+\cos \phi \cdot \cos \delta \cdot \cos \omega \\
\sin A=\frac{-\sin \omega \cdot \cos \delta}{\cos \alpha}
\end{array}\right.
$$

where $t_{b}$ is the local Beijing time, $\varphi$ is the local latitude, $\omega$ is the solar hour angle and $\delta$ is the Solar declination at that time.

From the formulas above, we can calculate the sun's direction in a certain latitude at a certain time,

$$
\stackrel{\mathrm{r}}{n}=\left(-\tan A, \frac{\varphi}{|\varphi|},-\tan \mathrm{h} \times \sqrt{\tan ^{2} A+1}\right) .
$$

\subsection{Figure the light intensity of a certain point as a source of the diffuse reflection.}

When the sunlight reach the trees, we take the layer of trees as a medium. So all the light must first pass to the tree layer, and then to the ground. As the same time, the tree layer receive sunlight and becomes a diffuse light source. Therefore, there are two parts of light that the tree layer receives, one is the light from the sun directly and the other part is the diffuse reflection from other trees(See the figure 1). Calculating the intensity of the illumination in the forest is finally the question of calculating the radiation intensity of all the diffuse light source(the tree layer).

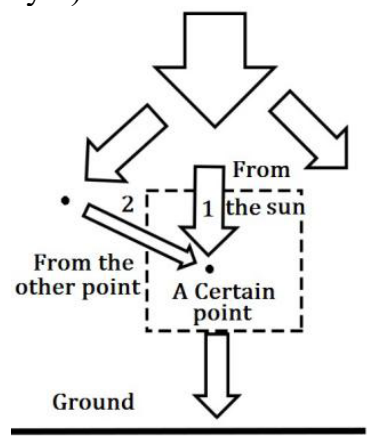

Figure 1. The light of a certain point.

Then we will discuss how to figure the light intensity of a certain point as a source of the diffuse reflection.

\subsubsection{The Light From The Sun Directly Makes A Source of The Diffuse Reflection}

The light intensity can be defined as the Lambert model. The intensity of diffuse reflected light is approximated to Lambert's law ${ }^{[2,3]}$, which can be expressed as follows,

$$
I_{1}=I_{0} k_{d} \cos \theta
$$

where,

$I_{1}$ is the intensity of diffuse reflection of the surface,

$k_{d}$ is the diffuse reflection coefficient,

$I_{0}$ is the intensity of the sunlight,

$\theta$ is the angle between the surface normal of the object and the direction of the sun. 


\subsubsection{The Diffuse Reflection From Other Point Makes A Source of The Diffuse Reflection}

A certain point catch the light from a other point consists 3 stages.

Stage 1: The sunlight shine the other point, and the other point becomes a source of the diffuse reflection. The light intensity can be expressed as,

$$
I_{1}=I_{0} k_{d} \cos \theta .
$$

Stage 2: A certain point shined by the diffuse reflection from the other point. The luminous flux that a certain point received can be expressed as,

$$
\Phi_{\text {in }}=I_{1}^{\prime} \Omega \text {. }
$$

Stage 3: A certain point becomes a source of the diffuse reflection. A certain point receive the diffuse reflection from the other point. These light minus the part absorbed by the leaves is the reflection luminous flux.

$$
\Phi_{\text {out }}=a_{l} \Phi_{\text {in }} \text {. }
$$

And the light intensity of a certain point because of the diffuse reflection can be expressed as,

$$
I_{2}=\frac{\Phi_{\text {out }}}{\Omega}=\frac{a_{l} I_{1} \Omega}{4 \pi} .
$$

\subsubsection{The light intensity of a certain point}

The light intensity of a certain point as a source of the diffuse reflection consists two parts. one is the light from the sun directly(like formula 4) and the other part is the diffuse reflection from other trees(like formula 7).So we can figure the light intensity of a certain point as a source of the diffuse reflection ${ }^{[3,4]}$.

$$
I_{\text {all }}=I_{1}+I_{2} \text {. }
$$

\subsection{The relationship of tree density, slope gradient and the diffuse reflection coefficient}

In the previous analysis, we used the diffuse reflection coefficient as a constant. However, in fact, there is a great relationship between the diffuse reflection coefficient and the density of the tree, the greater the tree density, the greater the diffuse reflection coefficient. In the third part we thus build the model A to discuss the relationship between the diffuse reflection coefficient and the density of the tree. And model B to discuss the relationship between tree density and slope gradient.

\subsubsection{Model A-- tree density and slope gradient}

In general, the greater the slope gradient, the greater the tree density. Initially, the density of the tree is not greatly affected by the change of the slope, but it falls down sharply at a certain slope and finally tends to 0 when the slope is high enough. The relationship between the two flat at first, then violent and flat again at last, so we use the logistic function to describe its changes.

$$
d_{t}=\frac{1}{1+e^{-b \times \cos \theta_{s}+c}},
$$

where $b$ and $c$ are constants, $\theta_{s}$ is the slope gradient.

\subsubsection{Model B-- the diffuse reflection coefficient and the density of the tree}

The greater the tree density, the greater the diffuse reflection coefficient, here we assume that the diffuse reflection coefficient is approximately proportional to the tree density.

So we can get a formula as follows,

$$
k=d_{t} .
$$

But the diffuse reflection coefficient has an upper limit instead of growing and growing with the increase of tree density. Therefore, we add a constraint factor to the formula (10)

$$
k=d_{t} \times e^{-f \times d_{t}},
$$

where $f$ is a constant, $d_{t}$ is the trees' density and $k$ is diffuse reflection coefficient.

Finally we chose the following parameters, and curves of $A$ and $B$ are shown as follows

We can find the density of the tree is an intermediate variable. And the relationship of slope gradient and the diffuse reflection coefficient also can be computed.

\subsection{The Complete Model}

Now we get the diffuse reflection model from formulas (4),(7),(8) and diffuse reflection coefficient model from formula (9),(11). Combining the two we can get a formula to determine the overall light intensity.

$$
\left\{\begin{array}{l}
I_{1}=I_{0} k_{d} \cos \theta \\
I_{2}=\frac{a_{l}}{4 \pi} \iint I_{1} \times \Omega d S . \\
I_{\text {all }}=I_{1}+I_{2}
\end{array}\right.
$$

The overall luminous flux can be obtained by an integral.

$$
\Phi=\int I_{\text {all }} d \Omega .
$$

Therefore, the intensity of the illumination which means the luminous flux per unit area of the ground can be expressed as follow

$$
E_{\text {gound }}=\frac{d \Phi}{d S}=\frac{\int_{0}^{2 \pi} I_{\text {all }} \times \Omega d \Omega}{S}=\frac{2 \pi I_{\text {all }}}{S} .
$$

\section{Numerical results}

\subsection{The Influence of Time and latitude}

As for the impact of the latitude and time on the direction of the sunlight, we control variable and divided the the question into two parts ${ }^{[2,3]}$.

Figure 2. The impact of time.

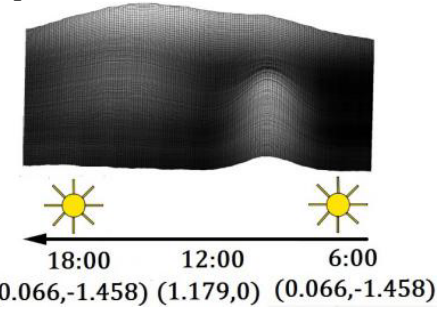


Figure 3. The impact of latitude.

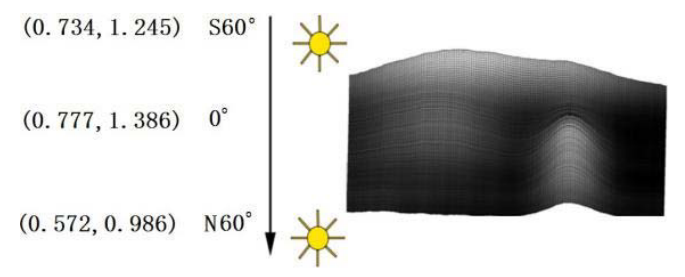

In the figure 2: A valley is shown in the centre of the figure. At a certain latitude, the sun rises at the east(the Figure 2), reach the middle of the figure at midday and finally reach the west(the Figure 2). From this figure, we can get the variations of the sun's direction at different time.

In the figure 3: A same valley is shown in the figure 3. At a certain time, the direction of the sunlight various with the latitude of the valley. The latitude of different valleys is marked in the figure, the position of the sun at midday is shown in the figure, too.

The solar elevation angle and the the solar azimuth angle are marked in the figure.

From the figure, we can get the variations of the sunlight's direction at different latitude. We will make a concrete analysis of this question after getting the result of the simulation.

\subsection{Terrain generation algorithm}

The actual terrain of mountainous region is complexity and variety. In order to evaluate the accuracy and practicality of our mode land take fully consideration of different situations as much as possible, we use terrain algorithm generate a random fractal terrain.

The flow-process figure of the terrain generation algorithm is shown as follows

Step 1. Generating boundaries;

Step 2. Internal expanding to generate random terrain;

Step 3. Superimposed mountain terrain(Please see figure 4).

Figure 4. Superimposed mountain terrain.

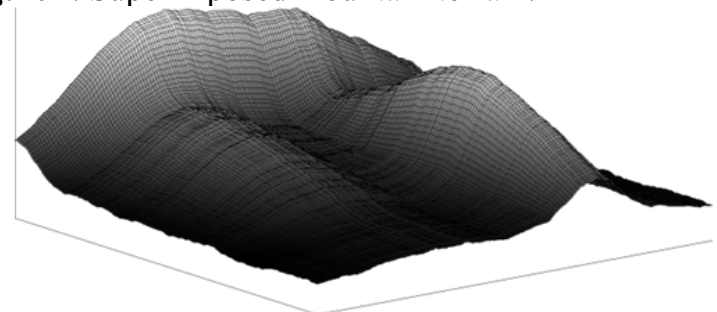

\subsection{The simulation results of the complete model}

Step1. Judge whether a certain point of the tree layer is under the direct light irradiation.

Contrast to the coordinates of the light rays and the actual mountain, you can know whether the light is blocked. The black line in the picture is the direction of sunlight. See the follow figure 5 .
Figure 5. Judging process.

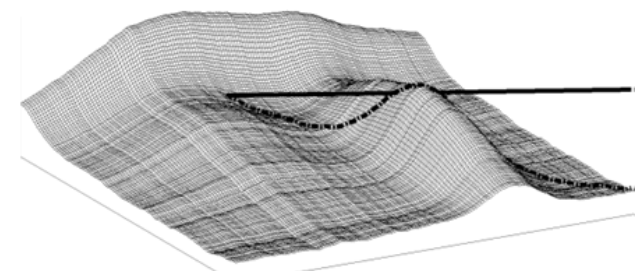

Step2. Judge which portions of the tree layer is in the direct sun.

Step3. In the first stage of diffuse reflection, if a certain point receive the sunlight directly, it can be regard as a diffuse reflection light source. The energy of the source are only comes from the sunlight directly.

Step4. In the second stage of diffuse reflection, every points of the tree layer receive light from other points more or less.

Step5. Therefore, every point of the tree layer becomes a diffuse reflection light source which contains two parts of light intensity. One comes from the sun directly and the other comes from other points of the tree layer.

Step6. Finally, the ground receive the overall light intensity from both the two stages of diffuse reflection. So the intensity of the illumination which means the luminous flux per unit area of the ground can be expressed in the following figures 6 .

Figure 6. The intensity of the illumination.

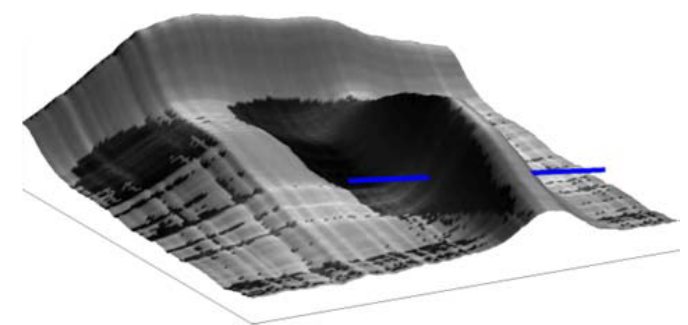

In this figure, the lighter the point seems to be, the greater the light intensity and the average luminescent of the area which receive the sunlight directly is 1.024 . The average luminescent of the area which not receive the sun's irradiation is 0.287 .

We can conclude that the valley is very dark because it is not exposed to the sunlight directly, but it is not without a little light. Finally, we have a comprehension simulation as follows:

Figure 7. Comprehension Simulation.

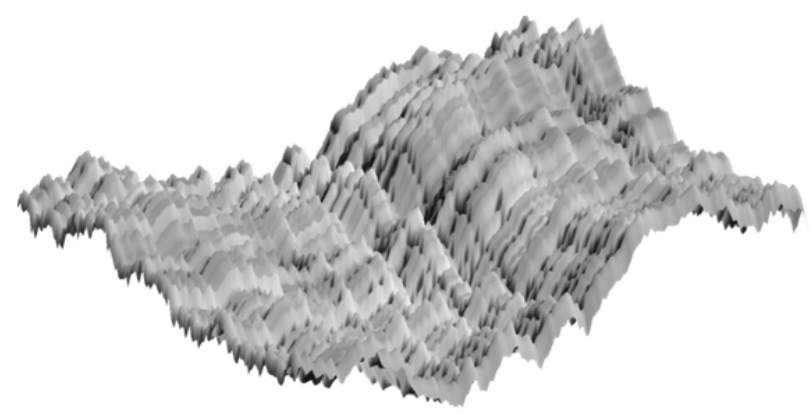




\subsection{Result Analysis}

The impact of the time and latitude on the direction of the sun are listed in the following figures.

Figure 8. The impact of the different times: 9:00,12:00,15:00 (the latitude is $0^{\circ}$ ).

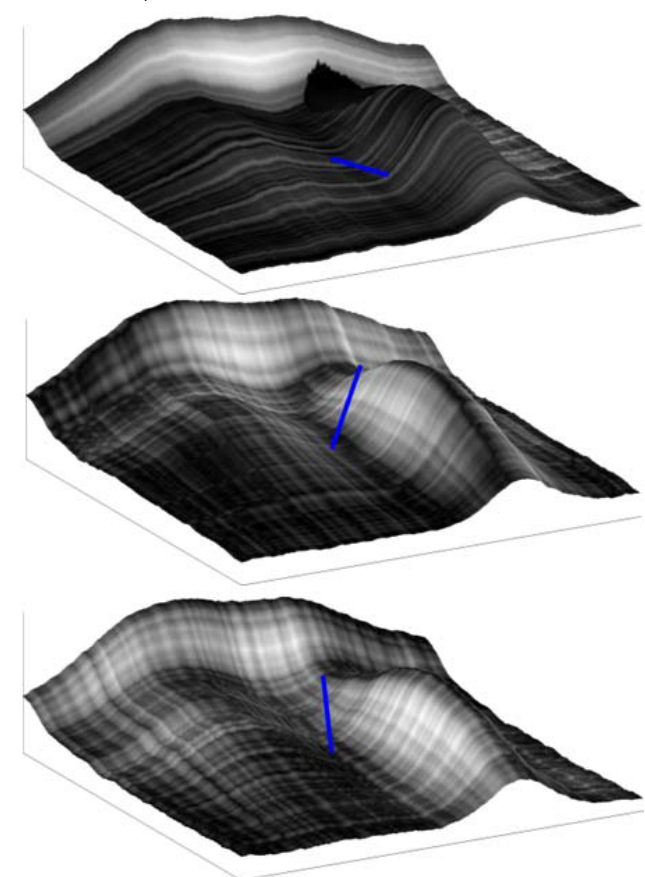

Figure 9. The impact of different latitudes: $\mathrm{N} 60^{\circ}, 0^{\circ}, \mathrm{S} 60^{\circ}$ (the time is 12:00).

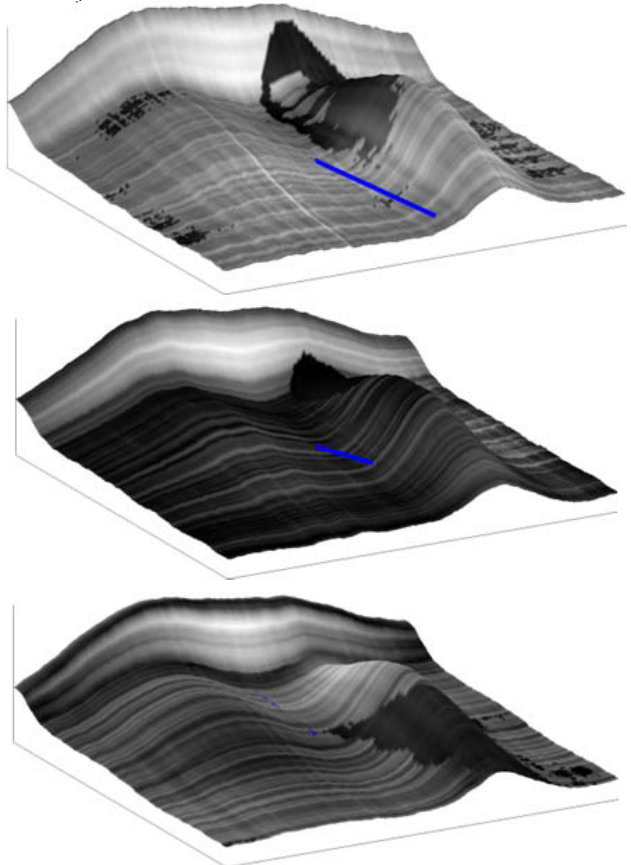

Apparently, the direction of the sun various with the time and the latitude and finally have impact on the intensity of illumination.

From figure 8 and 9, We can conclude that, the valley will be the brightest near the midday and quite dark in the early morning and at dusk. That is to say, brightness of the valley increases with the increase of the solar elevation angle.

Besides, the greater the absolute value of the latitude, the greater the light intensity of the valley. So we can know that the intensity of the illumination will be much lower near North and South Pole while the valleys close to the equator are more likely to receive adequate illumination.

\section{Conclusion}

In our paper, we change the question of the intensity of illumination into the effect under the diffuse reflection. This method not only simplify the question but also applicable in many conditions. Then we attempt to generate mountain randomly and study the illumination of the valley. What's more, our model can be used in many other conditions to evaluate the illumination situation. For instance, in the fields which vegetables are planted under fruit trees, there is a great relationship between the diffuse reflection of the trees and the light intensity the vegetables received. The model can be feasible to discuss whether the area is suitable for the vegetables.

However, The height of the tree is not in the consideration and we assumed that all kinds of terrains are evenly covered with trees. Moreover, most trees in the valley is not close enough to prevent the ground from the sunlight entirely. Especially in the sparse areas, large amounts of light go through trees and reach the ground directly. In this way, the real light intensity that the ground received will be much larger than our results, so does the intensity of illumination.

\section{References}

1. W.W. Zhang, X. You, G.M. Song, A Illumination model for landscape factors, Scienceof Surveying and Mapping, 34, 5 Sep.

2. C.Z. He, An Introduction to Mathematical Modelling, Chengdu, University of Science and Technology press, 1998.

3. G.Y. Ray, Mathematical Model of Lectures, Peking University Press, 1999.

4. Z.M. Zhu, H. Liu, S.M. Chen, The Design of Diffuse Reflective Free-Form Surface for Indirect Illumination With High Efficiency and Uniformity, IEEE P. J., 7, 3, 2015, 1600510. 